\title{
GLOBAL OPTIMIZED AND FULLY- INTEGRATED SHAPE OF THE NEW GENERATION OF SUPERSONIC AIRCRAFT
}

\author{
A. Nastase ${ }^{1}$ \\ ${ }^{1}$ Aerodynamics of Flight, RWTH, Aachen University, Germany (nastase @ lafaero.rwth- \\ aachen.de)
}

\begin{abstract}
The determination of the aerodynamical global optimized $(G O)$ shape of a flying configuration (FC), with respect of minimum drag at cruise, leads to an enlarged variational problem with free boundaries. The proposed strategy for the determination of the GO shape of the FC is the own developed evolutionary iterative optimum-optimorum (OO) theory, which chooses the GO shape among a class of elitary FCs. In the first step of its iteration, hyperbolic analytical potential solutions are used and the inviscid GO shape of FC is determined and is used as adequate surrogate FC's shape for the next step of iteration.The friction drag coefficient of the surrogate FC's shape is computed by using own developed analyticalnumerical hybrid solutions for the compressible full Navier-Stokes partial-differential equations and weak interactions with structure are performed. Up the second step of iteration, migrations in the drag functional, which is now the total drag (including friction) and in the constraints, including also those requested for structure purpose. Two new global optimized and fully-integrated Catamaran models are proposed for the supersonic transport aircraft and for the touristic aerospace aircraft, namely: one, which is optimized at cruising Mach number 2.2 and the second one, which is optimized at cruising Mach number 3.
\end{abstract}

Keywords: Hybrid solutions for compressible full Navier-Stokes partial-differential equations, Aerodynamical, global shape's optimization, Enlarged variational method, Supersonic flow, Catamaran supersonic aircraft

\section{INTRODUCTION}

The proposed strategy for the determination of the GO shape of FC is the own developed evolutionary iterative optimum-optimorum (OO) theory. The shape of the surface of FC is supposed to be piecewise approximated in form of superpositions of homogeneous polynomes in two variables with free coefficients. These coefficients together with the similarity parameters of the planform are the free parameters of the optimization. The used constraints are: the lift and the pitching moment coefficients are given, the Kutta condition along the subsonic leading edges is satisfied, the leading edges are sharp, the relative volumes of the wing and of the fuselage are given, the integration conditions (i.e. the wing and the fuselage have common tangent plane along their junction lines) are satisfied. The inviscid GO shape of the FC is 
determined in the first step of iteration and is used as adequate surrogate model for the next step of iterative OO theory. Up the second step of iteration the hyperbolical analytical start solutions for the optimization, used in the first step of iteration, are replaced with hybrid meshless numerical solutions for the three-dimensional full Navier-Stokes partial-differential equations (PDEs). The $\mathrm{OO}$ theory used here is an own, two times enlarged variational method, which is able to determine the aerodynamical GO shape of the FC and to include the friction effect in the computation of the total drag functional and in the GO shape.

The first enlargement consists in the determination of the inviscid GO shape of the FC (namely, the simultaneous optimization of its camber, twist and thickness distributions and also of its similarity parameters of the planform), which leads to an enlarged variational problem with free boundaries. An own OO theory was developed in order to solve this enlarged variational problem. The GO shape of the FC is chosen inside of a class of admissible FCs, which is defined by some common properties. Two FCs belong to the same class, if their surfaces are piecewise expressed in form of superpositions of homogeneous polynomes of the same maximal degree, their planforms are polygones, which can be related by affine transformations and which fulfill all the same constraints. A lower-limit hypersurface of the inviscid drag functional as function of the similarity parameters of the planform is defined:

$$
\left(C_{d}^{(i)}\right)_{\text {opt. }}=f\left(v_{1}, v_{2}, \ldots, v_{n}\right)
$$

Each point of this hypersurface is obtained by solving a classical variational problem with given boundaries (i.e. a given set of similarity parameters). The position of the minimum of this hypersurface, which is numerically determined, gives us the best set of the similarity parameters and the FC's optimal shape, which corresponds to this set, is at the same time the GO FC's shape of the class. This OO theory was used by the author for the inviscid GO shapes of three models, with respect to minimum drag, at the cruising Mach numbers 2, 2.2, and, respectively, 3. These models are: the delta wing alone Adela and, more recently, the fully-integrated wing-fuselage FCs Fadet I and Fadet II. Own developed three-dimensional analytical hyperbolical potential solutions, which have minimal singularities only along the singular lines and are written in integrated form, were used as start solutions for the performing of the inviscid GO of the shapes of these models. The aerodynamical performances of these models were checked in the trisonic wind tunnel of the DLR-Koeln. Very good agreements between the theoretical predicted and the measured lift and pitching moment coefficients were obtained.

The second enlargement of the variational method, used here, consists in the development of an iterative OO theory. The determination of this inviscid GO shape of FC, represents now the first step of iteration and is the surrogate model of the determination of the viscous GO shape. The friction drag coefficient of this surrogate model is computed by using new developed hybrid numerical solutions for the PDEs of the Navier-Stokes layer (NSL). Up the second step of iteration the total drag coefficient is the new functional of the iterative $\mathrm{OO}$ theory and the hybrid NSL's solutions are used. 


\section{THE INVISCID GLOBAL OPTIMIZATION OF THE SHAPE OF FLYING CONFIGURATION}

The determination of the inviscid GO shape of the integrated wing-fuselage FC, in order to have a minimum inviscid drag at cruising Mach number, represents the first step of an iterative viscous global optimization process, proposed here. This FC is treated like an equivalent integrated delta wing, fitted with two artificial ridges, which are located along the junction lines between the wing and the fuselage. The downwash $w$ on the thin component of this integrated FC is supposed to be continuous and expressed in the form of a superposition of homogeneous polynomes in two variables, namely:

$$
w=\tilde{w}=\sum_{m=1}^{N} \tilde{x}_{1}^{m-1} \sum_{k=0}^{m-1} \tilde{w}_{m-k-1, k}|\tilde{y}|^{k}
$$

The downwashes $w^{*}$ and $\bar{w}^{\prime *}$ on the wing and on the fuselage zone are expressed in form of two different superpositions of homogeneous polynomes :

$$
\begin{aligned}
& w^{*}=\tilde{w}^{*}=\sum_{m=1}^{N} \tilde{x}_{1}^{m-1} \sum_{k=0}^{m-1} \tilde{w}_{m-k-1, k}^{*}|\tilde{y}|^{k}, \quad \bar{w}^{\prime *}=\bar{w}^{*}=\sum_{m=1}^{N} \tilde{x}_{1}^{m-1} \sum_{k=0}^{m-1} \bar{w}_{m-k-1, k}^{*}|\tilde{y}|^{k} . \\
& \left(\tilde{x}_{1}=\frac{x_{1}}{h_{1}}, \quad \tilde{x}_{2}=\frac{x_{2}}{\ell_{1}}, \quad \tilde{x}_{3}=\frac{x_{3}}{h_{1}}, \quad \tilde{y}=\frac{y}{\ell}, \quad \ell=\frac{\ell_{1}}{h_{1}}, \quad v=B \ell, \quad B=\sqrt{M_{\infty}^{2}-1}\right)
\end{aligned}
$$

The coefficients $\tilde{w}_{m-k-1, k}, \tilde{w}_{m-k-1, k}^{*}$ and $\bar{w}_{m-k-1, k}^{*}$, together with the similarity parameter $v$ of the planform of the integrated GO of FC, are the free parameters of optimization and $\ell_{1}$ and $h_{1}$ are the half- span and the depth of this FC. The quotient $k=\bar{v} / v$ between the similarity parameters of the planforms of the wing and of the fuselage of this FC depends on the purpose of FC and is here considered constant.

The corresponding axial disturbance velocities $u$ and $u^{*}$ on the thin and thick-symmetrical components of the FC, obtained by the author, by using the hydrodynamic analogy of Carafoli, and the principle of minimal singularities (which fulfill the jumps along the singular lines) are, as in ${ }^{1}$ :

$$
\begin{gathered}
u=\ell \sum_{n=1}^{N} \tilde{x}_{1}^{n-1}\left\{\sum_{q=1}^{E\left(\frac{n-1}{2}\right)} \tilde{C}_{n, 2 q} \tilde{y}^{2 q} \cosh ^{-1} \sqrt{\frac{1}{\tilde{y}^{2}}}+\sum_{q=0}^{E\left(\frac{n}{2}\right)} \frac{\tilde{A}_{n, 2 q} \tilde{y}^{2 q}}{\sqrt{1-\tilde{y}^{2}}}\right\} \\
u^{*}=\ell \sum_{n=1}^{N} \tilde{x}_{1}^{n-1}\left\{\sum_{q=1}^{E\left(\frac{n-1}{2}\right)} \tilde{C}_{n, 2 q}^{*} \tilde{y}^{2 q} \cosh ^{-1} \sqrt{\frac{1}{v^{2} \tilde{y}^{2}}}\right.
\end{gathered}
$$




$$
\begin{gathered}
+\sum_{q=0}^{E\left(\frac{n-2}{2}\right)} \tilde{D}_{n, 2 q}^{*} \tilde{y}^{2 q} \sqrt{1-v^{2} \tilde{y}^{2}}+\sum_{q=0}^{n-1} \tilde{H}_{n q}^{*} \tilde{y}^{q}\left[\cosh ^{-1} M_{1}+(-1)^{q} \cosh ^{-1} M_{2}\right] \\
\left.+\sum_{q=0}^{n-1} \tilde{G}_{n q}^{*} \tilde{y}^{q}\left[\cosh ^{-1} N_{1}+(-1)^{q} \cosh ^{-1} N_{2}\right]\right\} \\
\left(M_{1,2}=\sqrt{\frac{(1+v)(1 \mp v \tilde{y})}{2 v(1 \mp \tilde{y})}}, \quad N_{1,2}=\sqrt{\frac{(1+\bar{v})(1 \mp v \tilde{y})}{2(\bar{v} \mp v \tilde{y})}}\right)
\end{gathered}
$$

The parameters of optimization are the coefficients of the downwashes $\tilde{w}_{i j}, \tilde{w}_{i j}^{*}$ and $\bar{w}^{*}$ and the similarity parameters of the planform of the entire FC and of the planform of the fuselage $v$ and, respectively, $\bar{v}$. For a given value of the similarity parameter $v$, the optimization of the shapes of its thin and thick-symmetrical components can be separately treated. The constraints of the inviscid GO shape's design are:

- the given lift and pitching moment coefficients and the Kutta condition along the subsonic leading edges of the thin FC component (in order to cancel the induced drag at cruise and to suppress the transversal conturnement of the flow around the leading edges, in order to increase the lift) and

- the given relative volumes of the wing and of the fuselage zone, the cancelation of thickness along the leading edges and the new introduced integration conditions along the junction lines between the wing and fuselage zone of the thick-symmetrical FC component (in order to avoid the detachment of the flow along these lines).

If the similarity parameter $v$ of the planform of FC is sequentially varied, a lower limitline of the inviscid drag functional of optimal FCs, as function of this similarity parameter $v$, is obtained by solving of classical variational problems with given planforms, for the corresponding value of the similarity parameter (for FCs with subsonic leading edges, $0<v<1$ ). The position of the minimum of this limit-line gives the optimal value of the similarity parameter $v=v_{o p t}$ and the corresponding optimal FC is, at the same time, the GO FC of the class. A hybrid analytical-numerical solution, according to the OO theory, is used. Each point of the lower limit-line of the inviscid minimum drag functional, for a given value of the similarity parameter $v$, is analytically determined, by solving of a corresponding linear algebraic system and the minimum of the lower limit-line is numerically obtained, as in ${ }^{1}$. The inviscid GO shape of the integrated model Fadet II, designed by the author, is presented in the (Fig. 1).

The lift and the pitching moment coefficients $C_{\ell}$ and $C_{m}$ of this model were measured in the trisonic wind tunnel of the DLR-Cologne, in the frame of some research contracts of the author, sponsored by the DFG.

The comparisons of theoretical and experimental determined lift and pitching moment coefficients of model Fadet II are presented in the (Fig. 2a,b). 


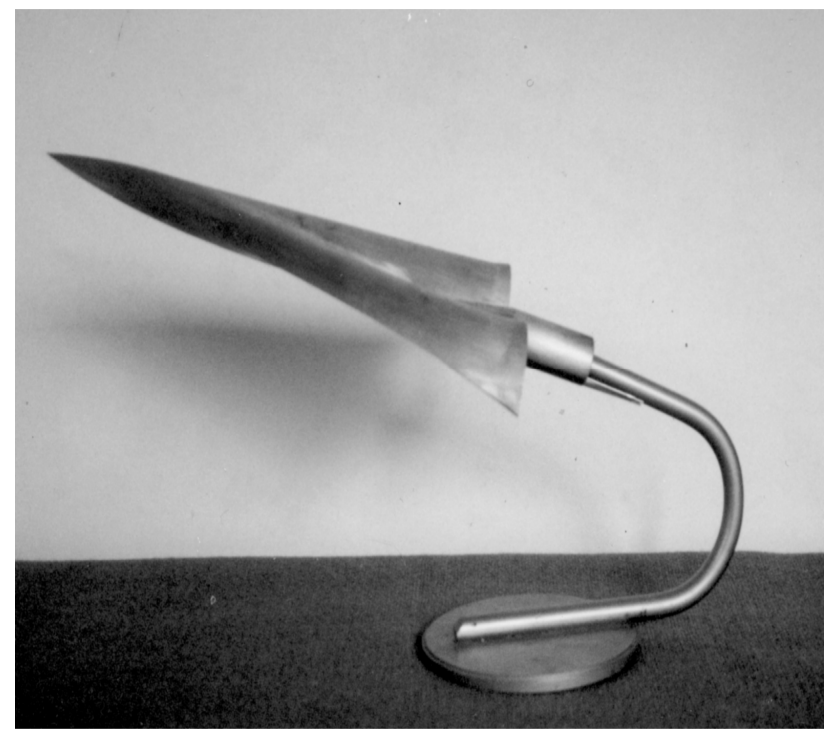

Figure 1. The Inviscid, Integrated and Global Optimized Shape of the Model Fadet II
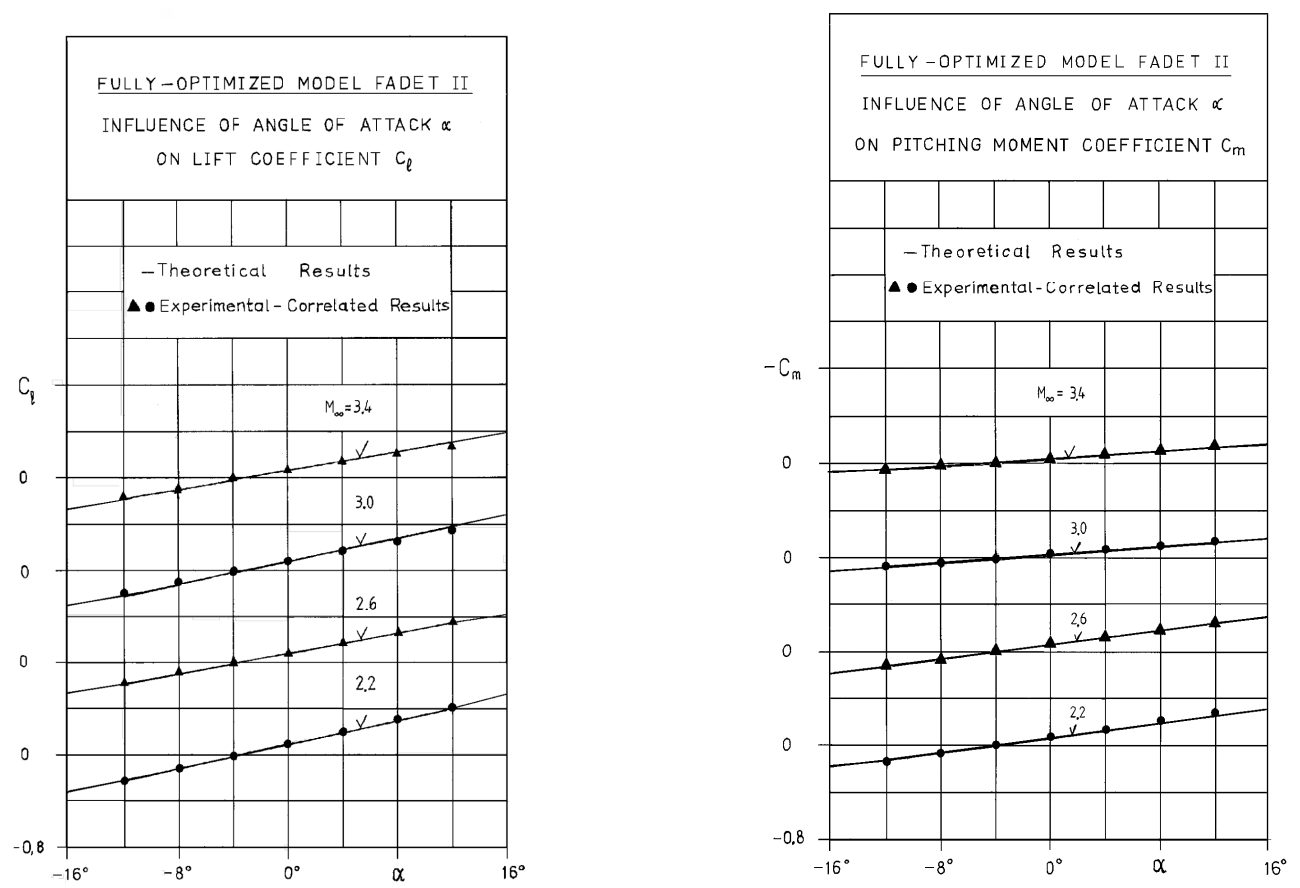

Figures 2a,b. The Theoretical and the Experimental-Correlated Lift and Pitching Moment Coefficients of Integrated, Global Optimized Model Fadet II.

The very good agreement between experimental and theoretical inviscid analytical hyperbolic solutions of the lift and pitching moment coefficients of FCs with subsonic leading edges, at moderate angles of attack (deduced by the author in closed forms), leads to the follo- 
wing remarks: the validity of the three-dimensional hyperbolical analytical potential solutions for the axial disturbance velocity with the chosen balanced singularities and the corresponding developed software for the computation of above aerodynamical coefficients are confirmed; the influence of friction upon these coefficients is neglectable; the flow is laminar, as supposed here and it remains attached in supersonic flow, for larger angles of attack than by subsonic flow. If the FC has sharp subsonic leading edges, is flattened enough and flies at moderate angles of attack, the flight with characteristic surface, instead of the flight with shock wave surface, is confirmed.

Solutions of the Navier- Stokes layer (NSL) are needed for the computation of the friction drag and for the performing of the viscous GO shape of FC.

\section{HYBRID SOLUTIONS FOR THE NAVIER-STOKES LAYER}

The proposed hybrid numerical solutions for the NSL's PDEs use the hyperbolical analytical solutions given before, as in 1,2 , twice, namely: as outer flow, at the NSL's edge (instead of the undisturbed parallel flow used by Prandtl in his boundary layer theory) and, secondly, the velocity's components are products between the corresponding potential velocities and polynomial expansions with arbitrary coefficients, which are used to satisfy the NSL's PDEs. Let us now introduce a spectral variable $\eta$, it is:

$$
\eta=\frac{x_{3}-Z\left(x_{1}, x_{2}\right)}{\delta\left(x_{1}, x_{2}\right)} . \quad(0<\eta<1)
$$

Hereby $Z\left(x_{1}, x_{2}\right)$ is the equation of the surface of the flattened FC and $\delta\left(x_{1}, x_{2}\right)$ is the NSL's thickness distribution. The spectral forms of the axial, lateral and vertical velocity's components $u_{\delta}, v_{\delta}$ and $w_{\delta}$, the density function $R=\ln \rho$ and the absolute temperature $T$ are here proposed, as in ${ }^{1,2}$, namely:

$$
\begin{gathered}
u_{\delta}=u_{e} \sum_{i=1}^{N} u_{i} \eta^{i}, \quad v_{\delta}=v_{e} \sum_{i=1}^{N} v_{i} \eta^{i}, \quad w_{\delta}=w_{e} \sum_{i=1}^{N} w_{i} \eta^{i}, \quad R=R_{w}+\left(R_{e}-R_{w}\right) \sum_{i=1}^{N} r_{i} \eta^{i} \\
T=T_{w}+\left(T_{e}-T_{w}\right) \sum_{i=1}^{N} t_{i} \eta^{i}
\end{gathered}
$$

Here $R_{w}$ and $T_{w}$ are the given values of $R$ and $T$ at the wall, $u_{e}, v_{e}, w_{e}, R_{e}$ and $T_{e}$ are the values of $u, v, w, R$ and $T$ at the NSL's edge, obtained from the outer inviscid hyperbolical potential flow, and $u_{i}, v_{i}, w_{i}, r_{i}$ and $t_{i}$ are their free spectral coefficients, which are obtained by fulfilling of the NSL's PDEs. The physical equation of ideal gas for the pressure $p$ and an exponential law of the viscosity $\mu$ versus $T$ are used:

$$
p \equiv R_{g} \rho T=R_{g} e^{R} T, \quad \mu=\mu_{\infty}\left[\frac{T}{T_{\infty}}\right]^{n_{1}} .
$$

Here are: $R_{g}$ and $T_{\infty}$ the universal gas constant and the absolute temperature of the undis- 
turbed flow and $n_{1}$ the viscosity exponent. The use of the density function $R=\ln \rho$ (instead of the density $\rho$ ), proposed here, of the formulas for $R, T, u, v, w, p$ and $\mu$, and of the collocation method allows the determination of the spectral coefficients of all the physical entities, namely: $R, T, p$ and $\mu$ only as functions of the spectral coefficients of the velocity's components, as in ${ }^{1-3}$. The spectral forms (6a-e) automatically satisfy the boundary conditions, at wall $(\eta=0)$. The boundary conditions at the NSL's edge are eliminated by fixing seven spectral coefficients of the velocity's components. If the spectral forms given in (6a-c) are introduced in the NSL's PDEs of impulse and the collocations method is used, the spectral coefficients $u_{i}, v_{i}$ and $w_{i}$ of the velocity's components are obtained by the iterative solving of a linear algebraic system with slightly variable coefficients. The analytical properties of the hybrid numerical NSL's solutions, proposed here, are the following: they have correct last behaviors, correct and balanced jumps along the singular lines (like subsonic leading edges, junction lines wing/fuselage and wing/ leading edge flaps etc.), according to the minimum singularities principle. Additionally, for the supersonic flow, the condition on the characteristic surface is, automatically, fulfilled.

\section{THE GLOBAL OPTIMIZED AND ALMOST FULLY- INTEGRATED CATAMARANS STA AND LEO}

The viscous iterative $\mathrm{OO}$ theory uses the inviscid hyperbolical potential solutions as start solutions and the inviscid GO shape of the FC, only in its first step of iteration. An intermediate computational checking of this inviscid GO shape of the FC is made with own hybrid solvers, for the three-dimensional NSL. The friction drag coefficient $C_{d}^{(f)}$ of the FC is computed and the inviscid GO shape is checked also for the structural point of view. A weak interaction aerodynamics/structure is proposed. In the second step of optimization, the predicted inviscid GO shape of the FC is corrected by including these additional constraints in

the variational problem and of the friction drag coefficient in the drag functional. The influence of friction in the total drag is important, namely: about $20 \%$ at angle of attack $\alpha=0^{\circ}$ and cruising Mach number $M_{\infty}=2$.

The iterative $\mathrm{OO}$ theory is a deterministic evolutionary theory, which has almost all attributes of the genetic algorithms like migration (in the drag functional and in the constraints), mutation (in the start CFD-solutions from hyperbolical potential solutions to NSL's solutions and from inviscid to total drag functional), crossover (the analytical hybridization creates numerical solutions with analytical properties). It can be useful for the determination of the aerodynamical GO of the shape of supersonic transport aircraft (STA) of second generation. In order to be more rentable, the new STA must have the double number of passengers as Concorde. There exist two classical solutions with one central non-integrated fuselage, one with greater diameter but shorter fuselage and the other with smaller but longer fuselage.

If the classical solution for STA with one cylindrical central non-integrated fuselage is used, the new STA has: either a fuselage with greater transversal section and same length, which produces more drag, more sonic boom and has more solicited structure but has less longitudinal bending, as the solution with a small fuselage with double length. If the STA has a thin long fuselage, which is prolonged in front, longitudinal instationary bending and roll moment occur, due to the alternate detachment of vortices on the long cylindrical fuselage. If the long fuselage is prolonged in the rear part, these undesired effects disappear, but the fuselage can break, if the landing gear is not totally extended. 
The solution proposed here by the author is the Catamaran supersonic transport aircraft, (STA) with twin smaller fuselages, which are obtained by cutting the classical small and long fuselage of the STA into two parts of the same length and relocating them one parallel to the other at the same horizontal level, in the central part of the wing, fully-integrated with the wing and almost all embedded inside the thickness of the wing (in order to have windows on both sides!).

The iterative OO theory was used for the determination of the GO shapes of fully- integrated Catamarans STA and LEO models, optimized at cruising Mach numbers $M_{\infty}=2.2$ and, respectively, $M_{\infty}=3$.

\section{CONCLUSIONS}

The Catamaran has some advantages, when it is compared with the STA with one, central, non-integrated fuselage, carrying the same number of passengers, at the same cruising Mach number:

- it flies with a shock-free surface;

- it has no sonic boom interference because it flies with one characteristic surface (the classical STA with one central non-integrated fuselage flies with two shock surfaces, one produced at the frontal part of the fuselage and the other at the roots of the wing and in their intersection zones, the sonic boom interference occurs);

- it has a better structural stiffness and increased lateral stability; because instead of one long fuselage there are two twin fuselages embedded in the wing, with half length;

- it needs less trim because the weight is better distributed and the pressure center and the center of gravity points are more closely together;

- it has a higher $L / D$, due to global optimization, full integration, of flattening and due to the fulfilling of the Kutta condition along its subsonic leading edges, which avoids the leading edges conturnements, which cancels the induced drag, destroys the leading edge vortices and increases the lift, not only at cruise but also for large ranges of Mach numbers and angles of attack, etc.

There are the reasons that I recommend the global optimized and full-integrated Catamarans shaped supersonic aircraft and space vehicles, which are like gliding birds shaped FCs, for the new generation of FCs.

\section{REFERENCES}

[1] Nastase A., "Computation of Supersonic Flow over Flying Configurations". Elsevier Oxford, UK, (2007).

[2] Nastase A., "Hybrid Navier-Stokes Solutions for Aerodynamical, Global Optimal Shape's Design”, Proc. of Int. Conf. EngOpt, Paper 750, Rio de Janeiro, Brazil, (2008).

[3] Nastase A., "Multipoint and Iterative Aerodynamical Global Shape's Optimization". Proc. of Evolutionary and Deterministic Methods for Design Optimization and Control, of EUROGEN 2011, Paper 30, CIRA, Capua, Italy (2011). 